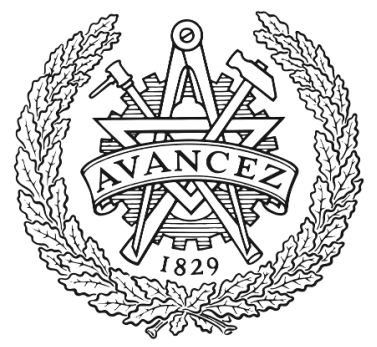

CHALMERS

UNIVERSITY OF TECHNOLOGY

\title{
Towards safe human robot collaboration - Risk assessment of intelligent automation
}

Downloaded from: https://research.chalmers.se, 2023-04-26 10:03 UTC

Citation for the original published paper (version of record):

Hanna, A., Bengtsson, K., Götvall, P. et al (2020). Towards safe human robot collaboration - Risk assessment of intelligent automation. IEEE International Conference on Emerging Technologies and Factory Automation, ETFA, 2020-September: 424-431.

http://dx.doi.org/10.1109/ETFA46521.2020.9212127

N.B. When citing this work, cite the original published paper. 


\section{Towards safe human robot collaboration - Risk assessment of intelligent automation}

\author{
Atieh Hanna \\ Research \& Technology Development \\ Volvo Group Trucks Operations \\ Göteborg, Sweden \\ atieh.hanna@volvo.com
}

\author{
Kristofer Bengtsson \\ Department of Electrical Engineering \\ Chalmers University of Technology \\ Göteborg, Sweden \\ kristofer.bengtsson@chalmers.se
}

\author{
Per-Lage Götvall \\ Research \& Technology Development \\ Volvo Group Trucks Operations \\ Göteborg, Sweden \\ Per-Lage.Gotvall@volvo.com
}

\author{
Mikael Ekström Senior member, IEEE \\ School of Innovation, Design, and Engineering \\ Mälardalen University \\ Västerås, Sweden \\ mikael.ekstrom@mdh.se
}

\begin{abstract}
Automation and robotics are two enablers for developing the Smart Factory of the Future, which is based on intelligent machines and collaboration between robots and humans. Especially in final assembly and its material handling, where traditional automation is challenging to use, collaborative robot (cobot) systems may increase the flexibility needed in future production systems. A major obstacle to deploy a truly collaborative application is to design and implement a safe and efficient interaction between humans and robot systems while maintaining industrial requirements such as cost and productivity. Advanced and intelligent control strategies is the enabler when creating this safe, yet efficient, system, but is often hard to design and build.

This paper highlights and discusses the challenges in meeting safety requirements according to current safety standards, starting with the mandatory risk assessment and then applying risk reduction measures, when transforming a typical manual final assembly station into an intelligent collaborative station. An important conclusion is that current safety standards and requirements must be updated and improved and the current collaborative modes defined by the standards community should be extended with a new mode, which in this paper is refereed to the deliberative planning and acting mode.
\end{abstract}

Index Terms-Human-robot Interaction, HRI, HRC, deliberation, safe interaction, operator education and training, safety standards, ISO/TS 15066.

\section{INTRODUCTION}

Installations of industrial robots are increasing globally every year [1], especially the use of collaborative robots (cobots) in small and medium enterprises. This is probably due to the benefit of easy to use and install as well as a reduced initial automation investment [2].

In the final assembly at an automotive factory, assembly stations are crowded with operators, product parts and tools,

This work has been supported by UNIFICATION, Vinnova, Produktion 2030 and ITS ESS-H Research School, The Knowledge Foundation. and the assembly process is often complex and in many cases hard to automate using traditional industrial robots. One reason is that these installations require necessary safety solutions including fences, which makes the combination of human and robot operations challenging. Therefore, there is a great interest in introducing automation in the form of collaborative robot systems where collaborative robots could help to relieve the human from the heavier and more laborious tasks and to free up his/her time for more value adding operations [3]. Although despite both industry and research are keen to develop the associated technologies with collaborative robots [4], cobots are underused [5]-[7] and are mostly deployed as traditional robots but without fences [8], mainly for handling tasks. They are not really used for collaboration as a true coworker, intelligent and collaborative, where they can increase both flexibility and quality.

Based on a previous study by the authors [9], where a manual assembly station was transformed into an intelligent collaborative automation system, the following requirements were identified for cobot systems:

1) The interaction between a cobot system and humans require that they can work side by side, co-actively or collaboratively, in a safe and efficient manner.

2) The cobot system must be fully aware of the human intention and adapt its behavior intuitively, while the human can access and understand system intention and information immediately without being distracted.

3) Smart tools that can be used by both humans and co-bot systems are needed to enable flexibility and robustness by interchangeability.

4) Managing variability, both of the product and the resources and their abilities, is required while planning and also in the control system. 
5) The communication and control system must be able to coordinate and control autonomous cobot systems as well as humans, whilst guaranteeing safe, efficient, and robust control, continuously re-plan the work.

Although all the requirements above are important when implementing intelligent and collaborative robot system, safety is a top priority. Manufacturers, integrators and users of robotic systems are required to ensure that the use of the system meets the available directives, standards and international specifications, where the harmonized standards play an important role in clarifying and specifying how to meet the safety requirements.

Based on the manual assembly station from the previous study [9], this paper focuses on the safety aspects of the intelligent cobot station with the goal of ensuring human safety while also achieving efficiency and flexibility. The possibilities and limitations of the available standards is evaluated in the paper by trying to use them during the design of the station. First, the mandatory risk assessment is performed for the station design and several possible risks are identified. Based on these risks, the proposed collaboration modes in current standards and specifications, are applied, trying to reduce the risks. However, the collaborative modes proposed by the standards do not differ notably from traditional automation and limit true intelligent robot human interaction. To improve the usefulness of the standards and specifications, we identified the need of a new mode, the deliberative planning and acting mode, to enable a deliberative interaction between human and robot system.

In the next section, the current safety standards are introduced. In Section III, the use case station is presented together with a description of the new collaborative station. The risk assessment of the collaborative station is presented in Section IV and in Section $\mathrm{V}$, the need for extended standards for intelligent collaborative systems is discussed.

\section{Regulation, SAFETy REQUiREMENTS AND STANDARDS}

There are many definitions of the relationship between humans and robot system, Wang et al. [10] classified them in four types, which are partly overlapping the nested classification suggested by De Luca et al. [11]:

- Coexistence is the simplest one when human and robot work side by side without overlapping other's workspace.

- Interaction occur when robot and human share the same workspace and communicate to complete a task in sequential order, without necessarily coordination of actions or intentions.

- Cooperation is possible when the parties have their own autonomy and strive to complete their task while sharing some of their resources.

- Collaboration goes further then the types mentioned already, is when human and robot directly interact, communicative or physical way, share intentions and coordinate to complete a complex task.
Depending on what type of relationship a specific operation has, the safety considerations will vary, which has an impact of the current standards. Robotic safety Standards are divided into three types. Type A provides general requirement for safety of machinery. Type B provides generic safety requirements and type $\mathrm{C}$ which provides more specific safety requirements have the highest priority and include references to type $\mathrm{A}$ and $\mathrm{B}$ standards.

The following standards are relevant and applicable for the type of automation system we are dealing with in this research:

- ISO 12100, Safety of machinery - General principles for design - Risk assessment and risk reduction [12].

- ISO 10218-1, Robots and robotic devices - Safety requirements for industrial robots - Part 1: Industrial Robots [13]. This part is intended for those who develop and manufacture the robot itself and its controller.

- ISO 10218-2, Robots and robotic devices - Safety requirements for industrial robots - Part 2: Robot systems and integration [14]. This part is aimed for those who integrate the robot system, including the robot, the endeffector and other equipment, devices products necessary to perform the required process.

- ISO/TS 15066, Robots and robotic devices - Collaborative robots [15]. This part supplements and builds upon the industrial robot safety standard ISO 10218. It provides additional guidance on design and risk assessment for collaborative robot application connected to the collaborative operation methods as well as defines biomechanical limits for power and speed limiting HRC applications. The specification is expected to become a standard in the near future [16].

According to the above safety standards and specifications, risk assessment is mandatory for all robotic applications. Its a comprehensive process that usually engage several stakeholders, from the integrator who is responsible for conducting the risk assessment, to the operator who will use the robot system. The process is iterative and begins with hazards identification, estimation and analysis of their severity and ends up with risk reduction.

According to ISO 12100 a risk is a function of its severity degree and the probability that the risk occurs. The probability is divided into three parts: how many people are affected, the occurrence of the risk and the opportunity to avoid or to limit the damage. Risk reduction principles in the standard are prioritized according to how effective they are at reducing risk. The most demanding approach is to reduce risk through inherently safe design. This is to be achieved by designing the risk so that it no longer exists. Second, the most difficult approach is to reduce the risk by providing protection, safeguarding, which prevents the risk from occurring or by protective measure to mitigate the risk so that it does not have the same serious consequences. If the two methods above are not possible to apply or an unacceptable risk remains, the last alternative is to inform about the risks, to train operators and staff, to use personal protective equipment and to warn where 


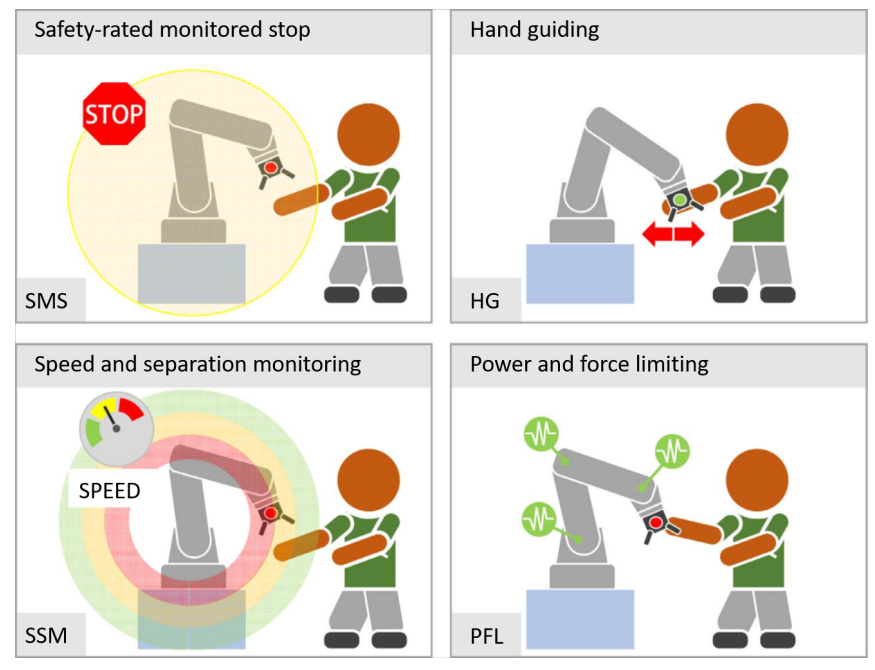

Fig. 1. Four Collaborative modes according to ISO 10218:2011, from Villani et al. [4]

the risk exists by setting up symbols in and/or around the area.

For collaborative applications additional risk may arise when robot and operator work side by side and share workspace. Therefore it is very important to eliminate the hazards and reduce the risks in the design phase by establishing the limits, the access and the clearance of the collaborative workspace, and ergonomics and human interface with the robotic system. According to ISO/TS 15066, task definition and task transition between collaborative and non-collaborative operations need to be carried out and be combined with the identified hazards related to robots, robot system and the application itself. Moreover, ISO/TS 15066 give clarification and guidance on how to apply one or a combination of several of the following collaborative operation modes, see Figure 1 from Villani et al., [4] to meet safety requirement and risk reduction when designing a collaborative application:

Safety Monitored Stop - This mode is used in applications where the robot system and human need to work side-by-side or to access the collaborative workspace, but not simultaneously. It is based on the robot system reaching a "protective stop" (Stop category 2 - a controlled stop with power left to the machine actuators) before an operator enters the collaborative area. The robot remains still until the operator leaves the area then it may resume automatically. Some collaborative robots are featured with motion limitation and function to achieve protective stop, but even traditional industrial robots can be used with complementary safety devices such as a safety camera or light curtain that monitor the workspace for any human entrance.

Speed and Separation Monitoring - This mode is an enhancement of the simpler Safety Monitored Stop mode where both operator and robot can act concurrently in the collaborative area. An area can be divided into a safe, a warning and a stop zone where the robot may move at higher speed when the operator remains in the safe zone, slow down when in the warning zone or stop when the operator is the stop zone. The robot (or external safety equipment) shall be equipped with safety-rated speed monitoring functions, safetyrated stop monitoring functions and safety-rated soft-axis and space limiting functions.

Hand Guiding - Is used when the operator needs to reprogram robot paths or position on the fly. It allows to transmit motion commands to the robot system via a handoperated device. This mode requires that the robot is equipped with safety-rated stop monitoring to ensure the safety of the operator entering the collaborative area and safety-rated speed monitoring to ensure that the robot is moved in a controlled speed where no additional hazards are introduced.

Power and Force Limiting - This mode is the "most" collaborative mode where the robot and the operator can have physical contact intentionally or unintentionally. It requires the robot system to have a passive and inherently safe design such as smooth surfaces and that the robot (or external safety equipment) is equipped with an active safety-related control system such as sensitive force monitoring functions that can dissipate collision forces and that do not violate determined force limits. Detailed information about guidance on force, speed, separation distance, biomechanical and contact limits and how to measure these can be found in ISO/TS 15066.

\section{THE USE CASE}

The use-case in this paper is a manual assembly station from a truck engine final assembly line. The engines are transported from station to station in a predetermined time slot on Automated Guided Vehicles (AGV), controlled by a factory control system, where the engine is set to a certain position and height. The material to be mounted on a specific engine are loaded by an operator from kitting facades located adjacent to the line, see Figure 2 . The kitting cart is manually pushed from the kitting area to the station and attached to the AGV just in time, where it will follow the AGV until all parts are consumed. Another operator is guided by instructions displayed on a screen to perform typical assembly tasks that require human precision and flexibility.

In the chosen station, the operator has to mount a heavy metal lock (ladder frame) on top of the engine, see Figure 2, insert several pipes, enter and tighten a large amount of bolts, and mount and tighten three oil filters, by using different tools that are suspended from the ceiling. The station is designed in a way that everything is within reach for the operator in a safe and ergonomically way. These kind of manual operations are monotonous and sometimes hard to finish within the dedicated time. Therefore the operators from adjacent stations can help each other in completing the all tasks within the cycle time.

Many tasks of the use case station are hard to automate and need an operators' excellent capability of performing flexible operations, such as pick and place of small part of different variants, tweaking and twisting pipes to get them in place, or keeping cables in order to connect them in tight spaces on the engine. However, to automate some of the operations would be beneficial from an ergonomic, flexibility, and quality perspective. To be able to automate the manual station, traditional 

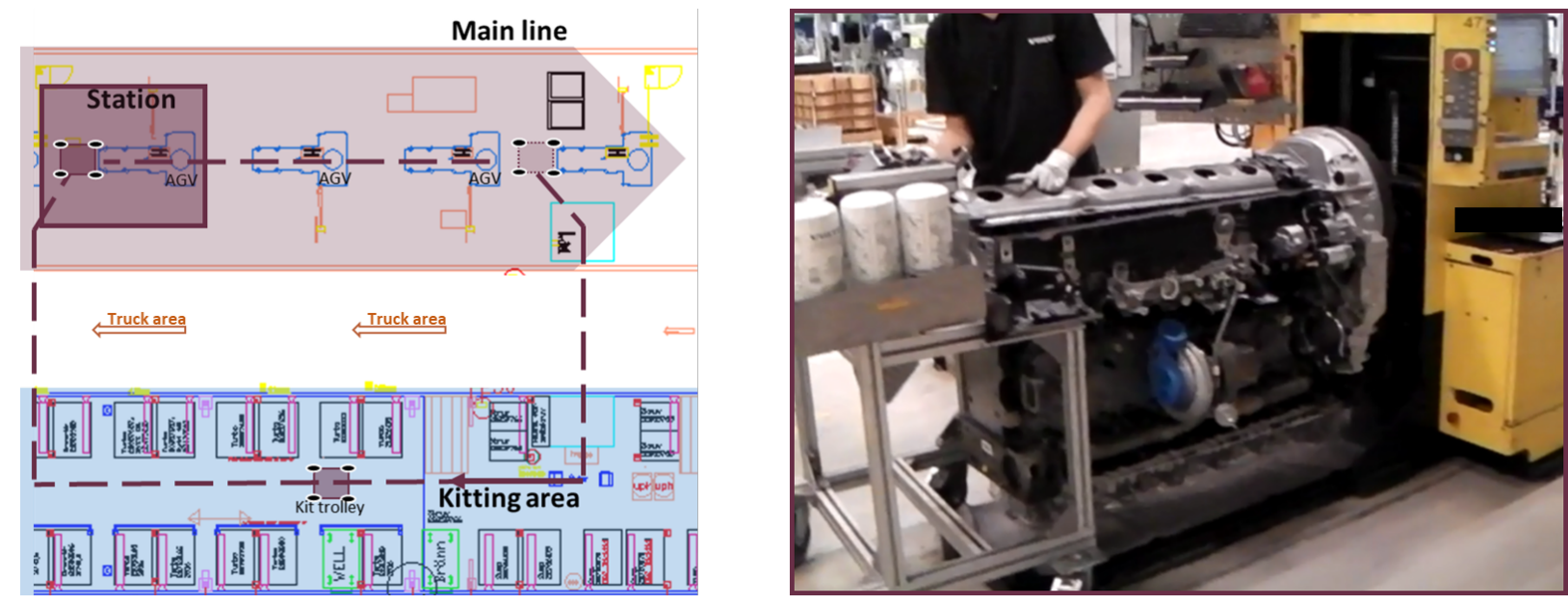

Fig. 2. Line layout with kitting area adjacent to mainline(left). The use case station where the operator is mounting the ladder frame on top of the engine(right)

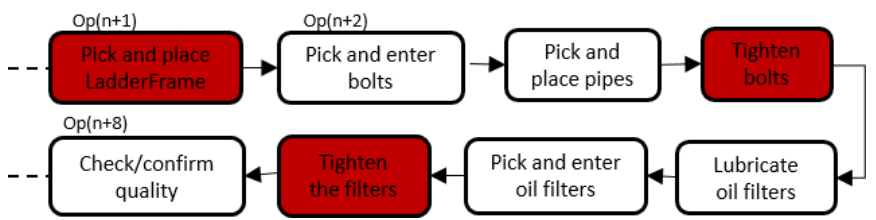

Fig. 3. Part of the operations performed sequentially by operator on the station

robots is not possible to use due to the restricted station space as well as the need to keep an operator, making this station a good use case for introducing collaborative robots. Some of the operations of use-case station was identified as suitable for collaborative or coexistence operations, and is shown in Figure 3.

\section{A. The collaborative station}

The conceptual layout for the transformation of the station can be seen in Figure 4. To increase flexibility an UR10e robot from Universal Robot, it is mounted on an autonomous mobile robot and operates at one side of the AGV that holds the engine, while the operator perform his/her task from the other side. With this separation, a natural barrier is obtained between robot and operator. The kitted material is transported just-in-time to the station by an Autonomous Transport Robot (ATR), which parks on the short side of the AGV to enable both the robot and the operator to reach the material.

To avoid unclear interaction between operator and robot, the station uses traditional visible marked boundaries (yellow and black stripes) on the floor to visualize the restricted area of the station for both active resources in the station, the robot system and the operator, and for other passive persons who may be near the station. The robot system has a predefined track to follow (blue dots) when entering and leaving the station boundaries.

An important aspect in the design was to design a solution that enable interchangeability between operator and robot so that in case the robot fails, the operator can replace the robot and finishes the task. Therefore the tools are adapted to be used both by the robot as well as the operator and wired from the ceiling right above the engine.

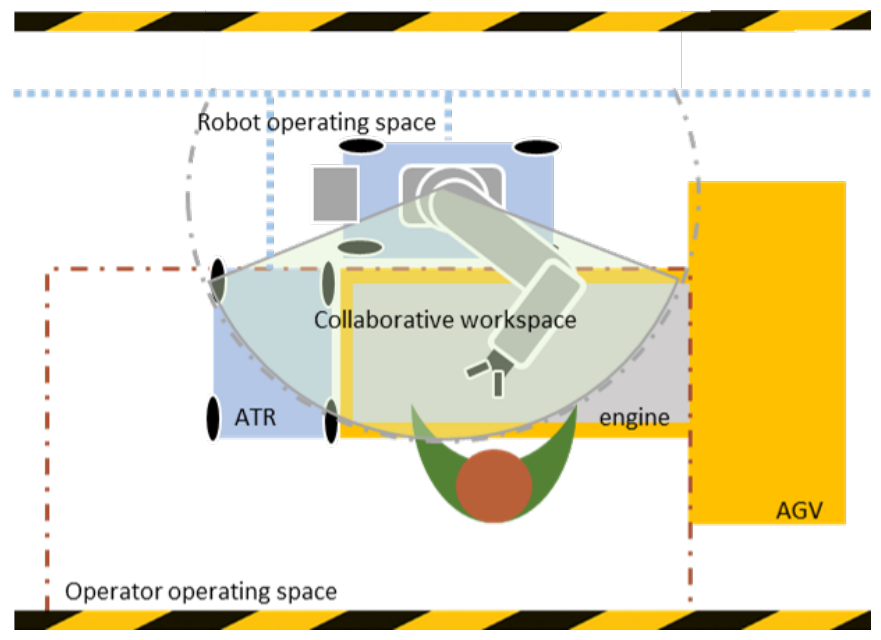

Fig. 4. The collaborative robot station of the use case

\section{B. Station tasks}

High level planning operations to be performed on the station are described below, and are also shown using a Sequence of operations(SOP) graph [17] in Figure 5, incl. allocation of tasks on robot (with prefix R), on operator(with prefix $\mathrm{O}$ ) or on both robot and operator (with prefix $\mathrm{O} / \mathrm{R}$ ) as well as the dependency between operations can be listed out. for example: the operation TightenBoltss has a precondition RobotReadyToTighten that must be fulfilled before the operation can be executed and when it is completed, post condition TightenBoltsLeftDone is updated.

1) The station cycle time begins when the precondition AGVReady is true and the AGV with the engine is at a predetermined position according to station configuration. Then, the operation ParkATR and ParkMobilRobot 
can be executed. Already when the robot comes to the station, it has an LFtool attached. Now the pick and place ladder frame operation can be executed by the robot together with the operator. A control algorithm adapts to the operator's ability to first lift and then navigate the heavy plate and place it on top of the engine.

2) It is only when the ladder frame is positioned on the engine and post conditioning LFOnEngine is fulfilled, that the operator proceeds with his operation PickAndPlacePipes, while the robot de-attaches from the LFtool and attaches to the nutrunner.

3) When the pre condition PipesOnEngine is fulfilled, the operator can execute PickAndEnterBolts. The robot can't execute TightenBolts before the pre condition NutrunnerReady and BoltsOnEngine are True. $\mathrm{O} *$ indicates that operator can execute the operation in case the robot fails or malfunctions.

4) When the robot is finished with TightenBolts on left side, the robot can continue with TightenBolts on right side, while the operator execute PickAndPlaceOilFilters to keep the cycle time.

5) When the pre condition TightenBoltsRightDone becomes true, the robot de-attaches from the nutrunner and attaches to the oil filter tool and proceed with executing TightenOilfilters, while the operator perform a quality check.

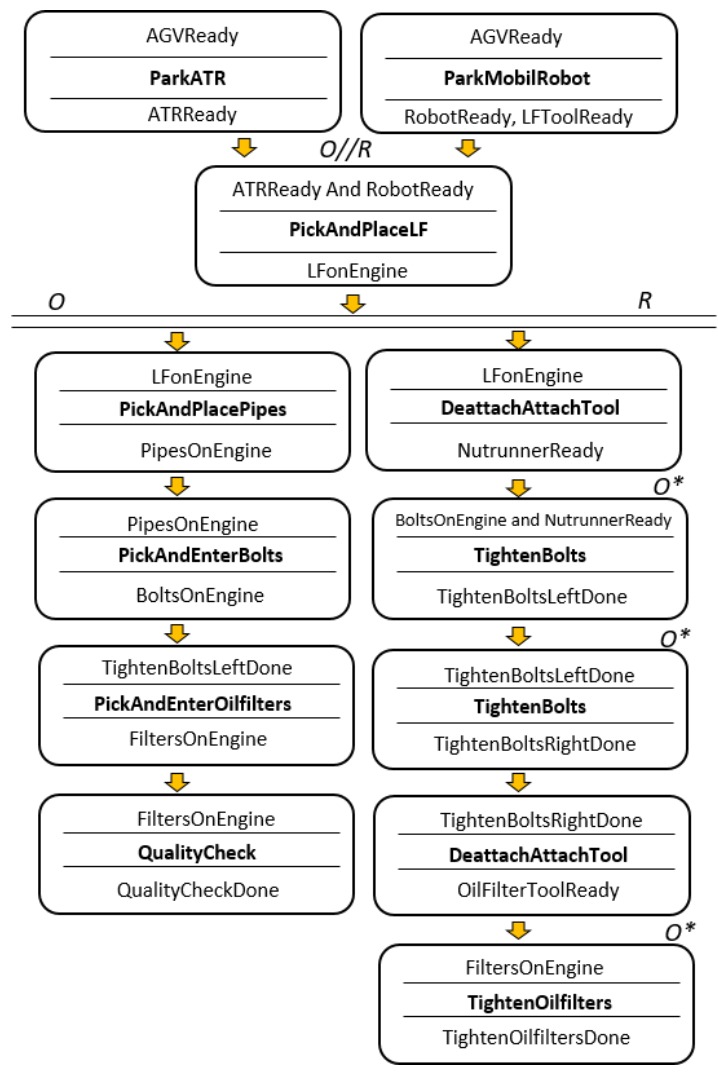

Fig. 5. Sequence of operations for the collaborative station

\section{Iterative design}

The design of the cobot station presented above, was in reality designed during multiple iterations together with the risk assessment. This iterative process started with the requirements related to the product, process and resources, when defining the application limitations and the conceptual layout. Consideration have been taken for what resources should and could perform the various operations for assembling the product and its variants, what components needed to be included and what resources e.g. tools, robots or operator needed to have the capabilities to perform the required tasks. In addition, which operator/engineer should have access to the station, what type of training and education is required and who is allowed to modify the configuration of the station. An important step was to define the collaborative area boundary and division between collaborative and non-collaborative tasks and dedicated operator/robot work-space in the station. However, since these design decisions is not the focus of this paper, the motivation of each design decision is left out of this paper due to brevity.

\section{RISK ASSESSMENT}

Based on the station design presented above, several hazards that may occur during the interaction between human and robot system was identified during the risk assessment. Some of the hazards are listed in Table I where a description of the hazard, each corresponding consequence, the level of collaboration, what related operations, and relevant performer are given.

For the level of collaboration, interaction and collaboration is used as defined in Section II, but a new human-robot relation is also used, called Coordination: human and robot system share a workspace and they communicates and coordinates their action and intention to complete a sequence of task.

Hazard 1 in Table I, is a common challenge for any type of automation system, where a hardware failure (or control error) make a robot drop what it is carrying, but can have greater consequences if it happens during a true collaborative operation. This failure can cause e.g. the heavy ladder frame to overturn on the operator's body or legs and injure him. Hazard 2 may occur when the robot and the operator share a workspace and execute their tasks in parallel, when e.g. a sensor failure makes the robot change its path unintentionally and hits the operator.

Hazard 3 is common if the control sequences are complex. It can happen in the case when the operator is picking and entering the bolts and the robot is tightening the bolts sequentially. If the sequence of the operator and the robot is not synchronized it may lead to that some steps are being missed, and that the robot tool may hit the operator while he/she is stressing to enter the bolts. Hazard 4 is similar to hazard 2 but is initiated by the operator who may not be aware of the robot state, which then moves in a direction not anticipated by the operator, probably due to communication failure, which lead to collision between the robot and the operator. 
TABLE I

HAZARDS IDENTIFIED

\begin{tabular}{|c|c|c|c|c|c|}
\hline No & Hazard & Consequence & Level of collaboration & Operations & Performed by \\
\hline 1 & $\begin{array}{l}\text { Robot loses grip on the heavy Lad- } \\
\text { der frame and drops it }\end{array}$ & Crushing & Collaboration & $\begin{array}{l}\text { Pick and place } \\
\text { ladderframe }\end{array}$ & Robot and operator \\
\hline 2 & $\begin{array}{l}\text { The robot does something unex- } \\
\text { pected and collides with the oper- } \\
\text { ator }\end{array}$ & Stroke to upper body & Interaction & $\begin{array}{l}\text { Any robot opera- } \\
\text { tion }\end{array}$ & Robot \\
\hline 3 & $\begin{array}{l}\text { The tool can hit the operator while } \\
\text { he/she pick and place the bolts }\end{array}$ & Sqeeze hands & Coordination & $\begin{array}{l}\text { Tighten bolt, pick } \\
\text { and place bolt }\end{array}$ & Robot \\
\hline 4 & $\begin{array}{l}\text { Operator unaware of system status } \\
\text { and unintentionally comes in the } \\
\text { way of the robot. }\end{array}$ & Collision & Interaction & $\begin{array}{l}\text { Any robot opera- } \\
\text { tion }\end{array}$ & Robot \\
\hline 5 & $\begin{array}{l}\text { The robot fails to attach to the } \\
\text { oil tool and the operator wants to } \\
\text { correct without signaling }\end{array}$ & Collision & Interaction & $\begin{array}{l}\text { Tighten oil filter, } \\
\text { troubleshooting }\end{array}$ & Robot \\
\hline
\end{tabular}

The final hazard 5, is another type of challenge since it relates to troubleshooting situations. It can occur e.g when the robot fails to attach to the oil filter tool, due to changed tool position and the robot stops. The operator may step in and try to correct without communicating with the system and suddenly the robot starts to move and collide with the operator.

\section{A. Risk reduction}

Next step is to assess the identified risks, determining if the risk level is acceptable or additional measures needs to be taken to control the hazards, reduce them or remove the unacceptable ones. The assessed criteria are: severity of injury $(\mathrm{s} 1, \mathrm{~s} 2)$, frequency of exposure to the risk $(\mathrm{f} 1, \mathrm{f} 2)$ and the possibility of avoiding the risk (p1, p2).

The outcome of the assessment is the required performance level (PLr) that the individual safety functions or safety measures, needed for minimising the identified risks, must comply with. In our application one or more identified hazard had the parameter severity of injury $=$ serious(s2), frequency and duration of exposure to the risk $=$ frequent and long time of exposure (f2) and possibility of avoiding the risk $=$ possible under specific conditions $(\mathrm{P} 1)$. Accordingly the resulted required performance level is $\mathrm{PLr}=\mathrm{d}$ which is the second highest level, see Figure 6

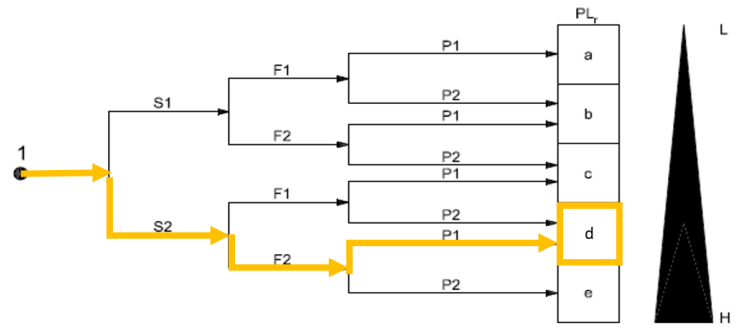

Fig. 6. Required performance level
For Collaborative robot systems, risk reduction is achieved through application design and workspace division (instead of separating human and robots by safeguards), and meeting the specific safety requirements and/or measures of one or a combination of the four collaborative operation modes defined by the standards.

To reduce risk 1 , it is possible to use both a mechanical safety design of the gripper and safety I/O with safety PLC that limits the actuation of the gripper when necessary. To reduce the other risks, we can possibly use several of the "built in safety" functions inherently designed in the robot UR10e [18] that comply with requirements specified by the four standards collaborative modes. The idea is that power and force limiting will always be active to reduce clamping and pressure exerted by the robot to reduce the risk level in case of collision between the robot and operator as in hazard 2 and 3. In those cases if any limit is exceeded a protective stop will be generated. In addition, to reduce hazard 3, speed limitation, safety-related soft axis and space limitation and TCP force and speed limitation can be applied, to ensure low speed of the robot while close to the operator, which can also make the operator feel more secure

In case of unintentional collision as for hazard 4, torque limiting can be used as well, to reduce high transient energy and impact forces. Hazard 5 is harder to handle, despite that the aforementioned functions are active, since the risk is still big enough if the operator is acting fast and is not aware of the system state and what is next robot sequence.

The disadvantages with these safety measure implementations are the limitation of speed of the robot, the use of safety zones, protective stops that need to be manually reset before resuming, etc, that makes the station very inefficient.

\section{B. Collaborative modes}

Apparently by using the aforementioned collaborative methods, by using a collaborative robot (UR10e) equipped with several safety functions, built-in force/torque sensor, configurable stopping time and stopping distance and by monitoring 
different parameters as speed and force, it seems that the majority of the risks can be reduced.

However, it is impossible to apply all these limiting strategies while meeting efficiency, flexibility and productivity requirements. The current collaboration modes of the standards and best practises can never enable the creation of a station that is safe and that can handle a large product variability, moving and exchangeable resources, handle errors and automatic recovery and online planning. We therefore suggest a new collaboration mode called Deliberation in planning and acting, that can support safe collaborative stations with intelligent and autonomous robot system that can include advanced control strategies.

\section{Deliberation In PLANNING AND ACTING}

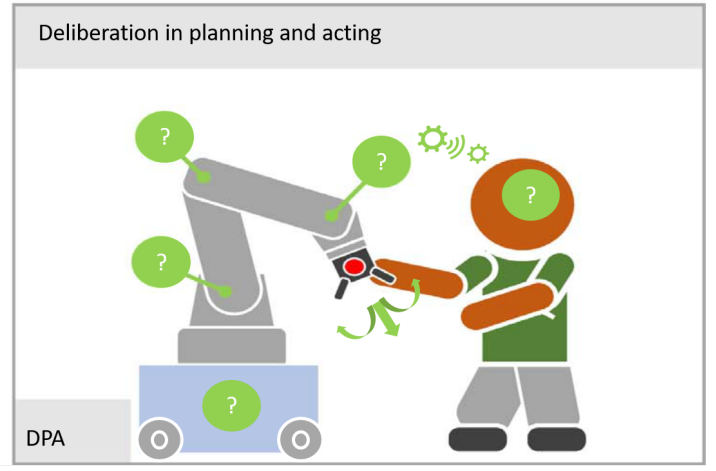

Fig. 7. Deliberation in planning and acting mode

In the deliberative planning and acting mode, represented in Figure 7 the operator and the robot system share the workspace, responsibilities, intentions, and they deliberate throughout the execution to reach an agreement of the plan for achieving a specific goal in an efficient and safe way. In order to implement this type of collaborative mode, the main component is a control system that can support the deliberation and that can execute the robots and other resources operations according to the agreed plan in a safe way [19]. However, some features from the other collaboration modes can be included, like adaptive power and force limiting functions, speed limiting functions, restricted zones and monitoring sensors.

A central requirement for this mode is that the operator should have a good understanding of the system and have received education and training to be able to operate in the workplace. The operator should be highly involved in designing the workplace, like defining sequence of operation, and he/she should have good knowledge of all resources capabilities to perform the different operations. Therefore it is very important that the robot system starts its operations by controlled access of a qualified operator.

A prerequisite for such a workplace is that an intuitive and bidirectional interface should be in place to enable efficient handshaking and checking, to indicate continuously the transition between the different collaborative tasks and to avoid erroneous action by either party. In case of changes to the plan, the operator and the robot system have to consult and deliberate with each other, via the intelligent human-in-theloop control system [20], before changing the agreed plan.

If the robot system anyway makes an undesired motion or action, the adaptive safety function should be active when executing plans with an increased risk. Since both the robot and the operator are aware about each other's state, they can agree on some operation where the robot can run at a higher speed while the operator understands what is safe or not and do not modify the agreed plan even if the operator can.

Including deliberation in planning and acting mode in our use case will make a difference in the risk reduction. For coordination operations, the deliberation mode will make the collaboration more efficient, since the operator and the robot are fully aware of each other's state and will deliberate in case they diverge from the plan. The risk of colliding with each other, as in hazard 2 and 3, becomes much lower as well due to the continuous synchronization of sequences in the deliberation moments. For hazards 4 and 5, the operator will not initiate a change in the sequence as without deliberate with the robot. The operator is also highly trained to know what actions that can not be taken without violating the plan [21].

\section{CONCLUSION}

In final assembly, where the environment is crowded and the process is complex, flexibility is a must when automating. By flexible automation we mean that the system must have a high degree of adaptation in order to handle the change over, both in volume variations, product and resource variability as well as to adjust production in time where we do not need to reprogram or re-validate after each change.

Apparently the balance between achieving flexible and economically sustainable automation solutions while ensuring safe interaction between the human and the robot system is challenging. The current collaboration methods for Humanrobot collaboration application design given by the harmonized standards are much similar to traditional automation and do not support implementation of intelligent and adaptive collaborative system in complex application. Therefore it is necessary to rethink safety and collaborative operation modes to enable the vision of working collaboratively with cobots and regarding them as truly co-workers.

For our study we have chosen a final assembly station and transformed it into an intelligent and collaborative station in several steps. In this paper, we have introduced the applicable standards and specifications for this type of automation. We have described the conducted risk assessment including possible risk reduction measures where we discussed the difficulty to include intelligence and maintain efficiency while meeting safety requirements. Based on the use case we have identified the need of a new collaborative method for deliberative and adaptive system where robot system and operator plan, deliberate, adapt and act together. This is important when updating future standards in the future. A prerequisite for this mode is a skilled operator with greater responsibility and experience, 
an intelligent and adaptive control system that can keep track of the current status in the application including the human, enabling smart algorithm and path planning as well as a robust communication.

\section{REFERENCES}

[1] I. E. summary world robotics 2017 industrial robots, Technical Report.International Federation of Robotics (IFR), 2017.

[2] RobotEnomics. https://robotonomics.wordpress.com/2016/01/11/thefacts-about-co-bot-robot-sales/, 2016.

[3] A. Cherubini, R. Passama, A. Crosnier, A. Lasnier, and P. Fraisse, "Collaborative manufacturing with physical human-robot interaction," Robotics and Computer Integrated Manufacturing, vol. 40, no. C, pp. 1-13, 2016.

[4] V. Villani, F. Pini, F. Leali, and C. Secchi, "Survey on human-robot collaboration in industrial settings: Safety, intuitive interfaces and applications," Mechatronics, vol. 55, pp. 248-266, 2018.

[5] W. He, Z. Li, and C. L. P. Chen, "A survey of human-centered intelligent robots: issues and challenges," IEEE/CAA Journal of Automatica Sinica, vol. 4, no. 4, pp. 602-609, 2017.

[6] P. Tsarouchi, A.-S. Matthaiakis, S. Makris, and G. Chryssolouris, "On a human-robot collaboration in an assembly cell," International Journal of Computer Integrated Manufacturing, vol. 30, no. 6, pp. 580-589, 2017.

[7] Å. Fast-Berglund, F. Palmkvist, P. Nyqvist, S. Ekered, and M. Åkerman, "Evaluating cobots for final assembly," Procedia CIRP, vol. 44, pp. 175 - 180, 2016, 6th CIRP Conference on Assembly Technologies and Systems (CATS).

[8] J. Saenz, N. Elkmann, O. Gibaru, and P. Neto, "Survey of methods for design of collaborative robotics applications-why safety is a barrier to more widespread robotics uptake," in Proceedings of the 2018 4th International Conference on Mechatronics and Robotics Engineering, 2018, pp. 95-101.

[9] A. Hanna, P.-L. Götvall, M. Ekström, and K. Bengtsson, "Requirements for designing and controlling autonomous collaborative robots systeman industrial case," Advances in Transdisciplinary Engineering, pp. 139144, 2018.

[10] L. Wang, R. Gao, J. Váncza, J. Krüger, X. Wang, S. Makris, and G. Chryssolouris, "Symbiotic human-robot collaborative assembly," CIRP Annals - Manufacturing Technology, vol. 68, no. 2, pp. 701-726, 2019.

[11] A. De Luca and F. Flacco, "Integrated control for phri: Collision avoidance, detection, reaction and collaboration," in 2012 4th IEEE RAS EMBS International Conference on Biomedical Robotics and Biomechatronics (BioRob). IEEE, 2012, pp. 288-295.

[12] SS-ISO 12100:2010 - Safety of Machinery - General principles of Design - Risk assessment and risk reduction. 2010. [Online]. Available: https://www.iso.org/obp/ui/iso:std:iso:12100:ed-1:v1:en

[13] SS-ISO 10218-1:2011 - Robots and robotic devices - Safety requirements for industrial robots - Part 1: Robots. 2011. [Online]. Available: https://www.iso.org/obp/ui/iso:std:iso:10218:-1:ed-2:v1:en

[14] SS-ISO 10218-2:2011 - Robots and robotic devices - Safety requirements for industrial robots - Part 2: Robot system and integration. 2011. [Online]. Available: https://www.iso.org/obp/ui/iso:std:iso:10218:2:ed-1:v1:en

[15] ISO/TS 15066:2016 - Robots and robotic devices - Collaborative robots. 2016. [Online]. Available: https://www.iso.org/obp/ui/iso:std:iso:ts:15066:ed-1:v1:en

[16] C. Franklin, "Standards revisions: robots and robotic systems," Control Engineering, vol. 64, no. 3, pp. 42-43, 2017.

[17] K. Bengtsson, P. Bergagard, C. Thorstensson, B. Lennartson, K. Akesson, C. Yuan, S. Miremadi, and P. Falkman, "Sequence planning using multiple and coordinated sequences of operations," IEEE Transactions on Automation Science and Engineering, vol. 9, no. 2, pp. 308-319, 2012.

[18] Universal Robot. [Online]. Available: https://www.universalrobots.com/e-series/

[19] E. Erös, M. Dahl, K. Bengtsson, A. Hanna, and P. Falkman, "A ROS2 based communication architecture for control in collaborative and intelligent automation systems," in Procedia Manufacturing, 2019.
[20] M. Dahl, E. Erős, A. Hanna, K. Bengtsson, M. Fabian, and P. Falkman, "Control components for collaborative and intelligent automation systems," in 2019 24th IEEE International Conference on Emerging Technologies and Factory Automation (ETFA). IEEE, 2019, pp. 378384.

[21] D. Romero, J. Stahre, T. Wuest, O. Noran, P. Bernus, Å. Fast-Berglund, and D. Gorecky, "Towards an operator 4.0 typology: a human-centric perspective on the fourth industrial revolution technologies," in Proceedings of the International Conference on Computers and Industrial Engineering (CIE46), Tianjin, China, 2016, pp. 29-31. 